\title{
Dilemma in the emergency setting: hypomagnesemia mimicking acute stroke
}

\author{
María Rico \\ Laura Martinez-Rodriguez \\ Davinia Larrosa-Campo \\ Sergio Calleja
}

Neurology Department, Central University Hospital of Asturias, Oviedo, Spain
Correspondence: María Rico Neurology Department, Central University Hospital of Asturias, José María Marcilla St 2C 7B Oviedo, 33006, Spain

Tel +34 407606369776

Email mricos@gmail.com
This article was published in the following Dove Press journal:

International Medical Case Reports Journal

II June 2016

Number of times this article has been viewed

Background: Stroke mimics may account for up to $30 \%$ of all acute stroke consultations. However, in the emergency setting, accurate diagnosis is not always possible.

Methods: Case report and review of the literature.

Results: A 73-year-old woman was admitted to the emergency department with acute aphasia and right hemiparesis. The National Institute of Health Stroke Score was 21, compatible with severe stroke, so she received thrombolysis. Laboratory testing demonstrated severe hypomagnesemia. She had been taking proton pump inhibitors for years and neuroimaging did not demonstrate signs of acute ischemic disease. After correcting the metabolic alterations with intravenous and oral supplemental magnesium, the patient was discharged asymptomatic. No further episodes have been registered to date.

Conclusion: Hypomagnesemia might cause acute neurological symptoms that could be confused with stroke. A careful history is essential for diagnosis but suspicion of stroke mimic should not prevent tPA administration.

Keywords: hypomagnesemia, stroke mimic, aphasia, stroke

\section{Background}

Stroke mimics (SMs) are rather frequent. An accurate diagnosis is essential not only to ensure proper treatment but also because misdiagnosis can lead to aggressive therapies with possible complications. On the other hand, the limitation of time and diagnostic tools in the emergency room add to the challenge. Should we refrain from administering thrombolytic therapy on the basis of a possible but unproved SM?

\section{Case report}

A 73-year-old woman with a medical history of hypertension, dyslipidemia, and active smoking presented with aphasia and right hemiplegia. According to her relatives, the symptoms started suddenly at $11.30 \mathrm{am}$. The Extrahospital Emergency Team evaluated her at home and Stroke Code was activated. The patient arrived at our hospital at $12.15 \mathrm{pm}$. She was apyretic on admission, with normal cardiorespiratory and gastrointestinal examination findings. Her blood pressure was 180/91 $\mathrm{mmHg}$. Neurological examination identified a receptive and expressive dysphasia, left gaze deviation, right hemianopia, mild right facial paresis, and moderate rightsided weakness. The National Institute of Health Stroke Score was 21, indicating a severe left hemispheric stroke. 
Upon further interrogation, her family described a history of anorexia and nausea for several previous weeks. Previous to the onset of focal neurological symptoms, she had not complained of headache nor had presented with fever.

The patient's regular medication included omeprazole 20 $\mathrm{mg}$ od, aspirin $100 \mathrm{mg}$ daily, atorvastatin $40 \mathrm{mg}$ daily, propranolol $40 \mathrm{mg}$ daily, irbesartan $150 \mathrm{mg}$ daily, venlafaxine $75 \mathrm{mg}$ daily, propafenone $150 \mathrm{mg}$ daily, methylprednisolone $4 \mathrm{mg}$ daily, calcium, and calcifediol. She also had macular degeneration, depression, fibromyalgia, essential tremor, and had undergone a hemithyroidectomy several years before. Also, she had suffered a first episode of stroke 2 years before.

Routine tests within the Stroke Code protocol included the following studies. Hemogram showed normal levels of hemoglobin, white blood cells, and platelets. There were no alterations in the coagulation screening. She had a potassium level of $3 \mathrm{mmol} / \mathrm{L}$ and glucose was $218 \mathrm{mg} / \mathrm{dL}$. Renal function was normal. No acute or chronic lesions were present in the brain computerized tomography (CT) (Figure 1A). CT angiography did not reveal any evident thrombus in proximal intracranial vessels (Figure 1B). Perfusion CT showed no volume or medium transit time alterations (Figure 2).

Suspecting fragmentation of the original thrombus with obstruction of multiple distal vessels, thrombolysis with 54 $\mathrm{mg}$ of intravenous alteplase was administered (dosage of 0.9 $\mathrm{mg} / \mathrm{kg}$ ). Despite the normal finding in the neuroimaging, there was no indication at that time of an alternative cause for the symptoms and the acute onset as well as previous history of cardiovascular risk factors prompted the decision to treat.

Extensive laboratory tests were performed after admission. Blood test revealed magnesium $0.10 \mathrm{mmol} / \mathrm{L}(0.66-0.99)$,

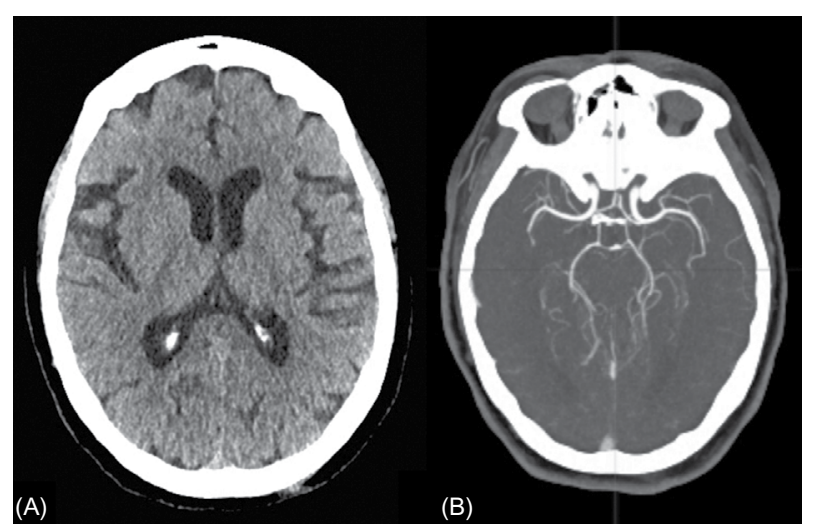

Figure I Neuroimaging in the emergency department

Notes: (A) CT brain scan shows the absence of hemorrhage or previous ischemic brain lesions. (B) CT angiography demonstrating normal contrast filling of the intracranial vessels.

Abbreviation: CT, computerized tomography. calcium $2 \mathrm{mmol} / \mathrm{L}(2.20-2.55)$, phosphorus $0.82 \mathrm{mmol} / \mathrm{L}$ (0.87-1.45), and iron $26 \mu \mathrm{g} / \mathrm{dL}(37-145)$. The rest of the screening was normal.

A second CT scan 24 hours after treatment with tPA was normal, but later brain magnetic resonance imaging (MRI) showed a convexal subarachnoid hemorrhage in the right occipital lobe, not present in the previous images, inconsistent with the initial symptoms and that went clinically unnoticed (Figure 3). No signs of acute ischemic damage were present in diffusion MRI sequences.

Regarding the underlying cause for the hypomagnesemia, the patient had no previous history of diarrhea or malabsorption problems and neither was she taking diuretics or any other drugs that could explain a urinary loss of magnesium, but she had been taking omeprazole for years.

Treatment with intravenous magnesium followed by oral supplements was administered. During the following days, the dysphasia, right hemianopsia, and hemiparesis slowly resolved, with remaining slight disorientation that also progressively disappeared. At discharge, she presented magnesium levels of $0.70 \mathrm{mmol} / \mathrm{L}$ and calcium $2.42 \mathrm{mmol} / \mathrm{L}$.

She was diagnosed with focal neurological symptoms secondary to severe hypomagnesemia; omeprazole treatment was stopped and no additional events have been reported to date. Full written consent was given by the patient for publication of the data included in this report. Ethics approval is not required by the institutional review board of Central University Hospital of Asturias for case reports.

\section{Discussion}

Between $6.5 \%$ and $30 \%$ of patients suspected of having an acute stroke in emergency departments are later diagnosed with SM..$^{1,2}$ Seizures, encephalopathy, syncope, and migraine are the most common causes and the most common referral symptom is aphasia. ${ }^{3,4}$

Among toxic/metabolic causes for SM hypoglycemia, hypo- and hypernatremia and hepatic encephalopathy are the best known, but hypomagnesemia may be responsible for several neurological manifestations, such as seizures, vertigo, nystagmus, encephalopathy, and less frequently focal deficits. ${ }^{5,6}$

The most common causes of hypomagnesemia are bladder and bowel loss, but the association between long-term use of pump proton inhibitor drugs and hypomagnesemia has been known for a few years now. ${ }^{6,7}$ Most typical symptoms are generalized weakness, anorexia, hypokalemia, hypocalcemia, and positive Trousseau and Chvostek signs. ${ }^{8}$ However, hypomagnesemia has been associated with a variety of neurological symptoms, including reversible mental status 


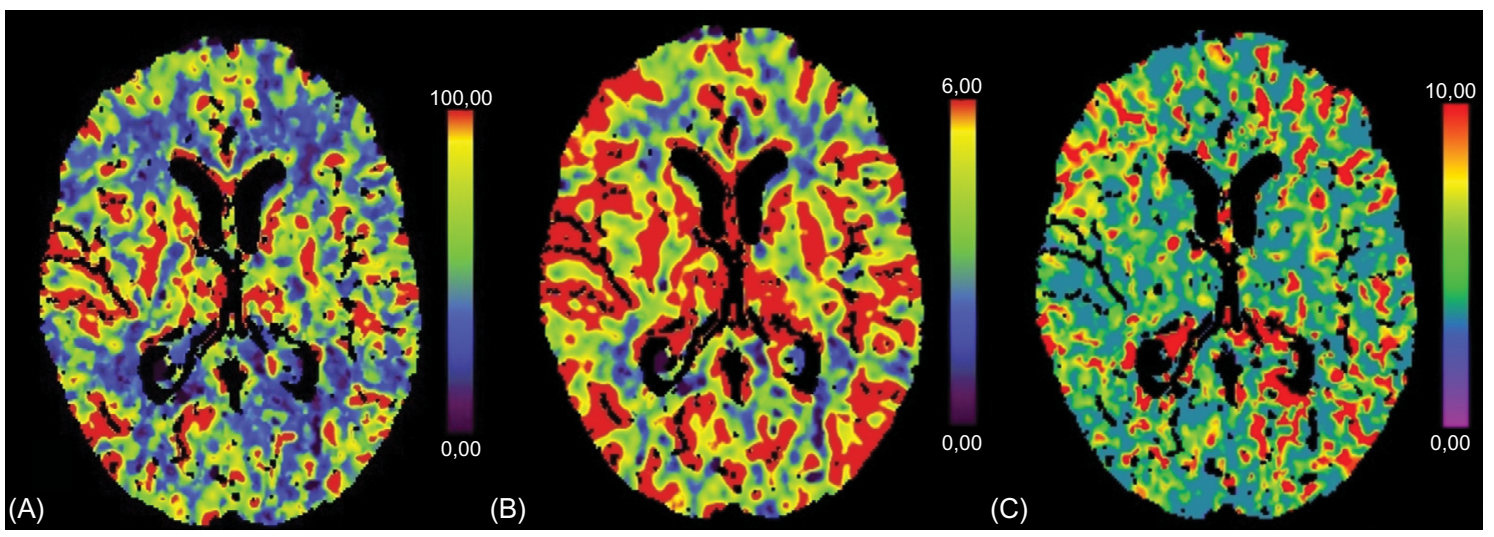

Figure 2 CT perfusion scan during the acute phase.

Notes: No asymmetries between both hemispheres are present in the cerebral blood flow (A), volume (B), or mean transit time (C) sequences.

Abbreviation: CT, computerized tomography.

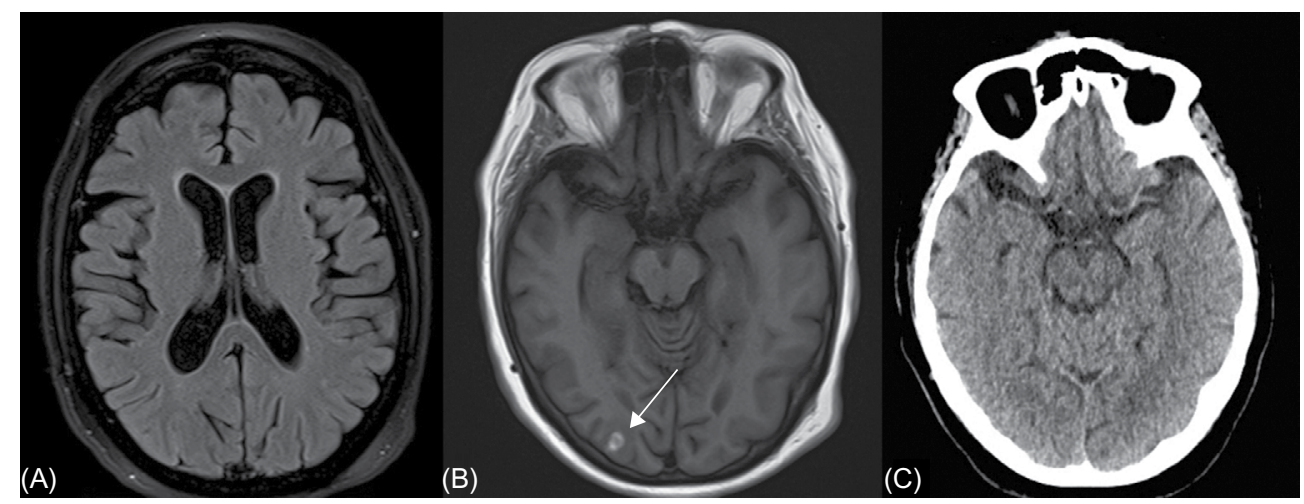

Figure 3 Post-thrombolysis 3T MRI.

Notes: (A) DWI-MRI sequence shows the absence of acute damage to the brain. (B) TI-weighted MRI sequences demonstrate the presence of a small acute subarachnoid hemorrhage in the right occipital lobe (arrow), not present in the emergency CT scan upon admission (C).

Abbreviations: DWI-MRI, diffusion-weighted imaging MRI; MRI, magnetic resonance imaging; CT, computerized tomography; $3 \mathrm{~T}$ MRI, 3 tesla resolution magnetic resonance imaging.

changes, seizures, and movement disorders. ${ }^{9}$ Among the focal manifestations, the most reported are cerebellar symptoms due to endothelial malfunction, presenting with reversible abnormalities on neuroimaging similar to those of posterior reversible encephalopathy syndrome. ${ }^{9-11}$ There is, however, a case of a 3-year-old boy who presented with aphasia in the context of severely low levels of serum magnesium. ${ }^{12}$ The history of proton pump inhibitors usage, prodromal symptoms of anorexia and nausea, low blood potassium levels, and normalcy of the perfusion imaging should have made us suspect that an SM was present in this case. However, in the emergency setting, these details went unnoticed and although magnesium blood levels could have probably been measured within the time-window, performing the test was not considered at the time.

Alternative diagnoses were considered in this patient, including other metabolic imbalances, seizures, encephalitis, and hypertensive encephalopathy. Glucose levels and liver function were normal. Although the symptoms could have been triggered by seizures, there were no signs of epileptic activity on electroencephalogram. Also, the patient had no fever or laboratory alterations that could suggest an infectious cause. Finally, although blood pressure was high on admission, it spontaneously reversed to normal levels without treatment during the stay in the emergency department and no signs of edema were found on CT scan, both going against hypertensive encephalopathy as a cause. A reversible vasoconstrictive syndrome was ruled out as a cause because of the normal angiographic sequences and perfusion imaging performed upon admission and the absence of headache. The presence of a right-sided occipital convexal hemorrhage on MRI was interpreted as a complication of treatment with thrombolytics rather than as the primary cause for the symptoms, as it was not present in the CT scan performed in the emergency department and inconsistent with the patient's symptoms of aphasia and right-sided hemiparesis. Also, although it could suggest the presence of an underlying vasoconstrictive syndrome, the angiographic sequences and perfusion imaging upon admission were normal. 
A low magnesium serum level as cause for SM, although rare, is not unheard of. The problem with SM lies in the limitations of its diagnosis in the emergency setting and, even more, in the implications if the correct treatment is withheld due to misdiagnosis. We could argue that when no anomalies are present on the perfusion CT scan, aborting the Stroke Code may be a good call, but it should be taken into account that the access to this technology is limited to tertiary stroke centers and that not all the hospitals where thrombolysis is administered have at their disposal. Furthermore, there are cases where despite normal CT perfusion imaging, or even diffusion-weighted imaging-MRI, the underlying cause is still a stroke. ${ }^{13,14}$ It is in these circumstances that the risk-benefit balance must come into play.

We should suspect SM in younger patients without any risk factors, especially if the neurological deficit is mild, but there are also cases such as ours in which the patient is older, has several cardiovascular risk factors, and presents with severe deficit. ${ }^{2,4}$ Is in these cases the mere suspicion a good enough reason not to treat? The most feared complication of intravenous tPA administration is bleeding. Although admittedly the risk for systemic hemorrhage still exists, some sources indicate that it may be even lower in SM patients than in stroke patients. ${ }^{15-17}$ On the other hand, the potential benefit of administering the treatment is well known. ${ }^{18}$

\section{Conclusion}

Hypomagnesemia may be the cause for neurological symptoms, including acute focal deficit that presents as an SM. A careful history and well-timed testing are essential for an accurate diagnosis.

SMs may account for up to $30 \%$ of all acute stroke consultations. Younger age, mild neurological deficit, and absence of risk factors should make us suspicious. However, except for contraindication or confirmed alternative diagnosis, t-PA administration should be favored.

\section{Disclosure}

The authors report no conflicts of interest in this work.

\section{References}

1. Forster A, Griebe M, Wolf ME, Szabo K, Hennerici MG, Kern R. How to identify stroke mimics in patients eligible for intravenous thrombolysis? J Neurol. 2012;259(7):1347-1353.

2. Merino JG, Luby M, Benson RT, et al. Predictors of acute stroke mimics in 8187 patients referred to a stroke service. J Stroke Cerebrovasc Dis. 2013;22(8):7.

3. Tobin WO, Hentz JG, Bobrow BJ, Demaerschalk BM. Identification of stroke mimics in the emergency department setting. $J$ Brain Dis. 2009;1:19-22.

4. Brunser AM, Illanes S, Lavados PM, et al. Exclusion criteria for intravenous thrombolysis in stroke mimics: an observational study. $J$ Stroke Cerebrovasc Dis. 2013;22(7):1140-1145.

5. Magauran BG Jr., Nitka M. Stroke mimics. Emerg Med Clin North Am. 2012;30(3):795-804.

6. Martin KJ, Gonzalez EA, Slatopolsky E. Clinical consequences and management of hypomagnesemia. JAm Soc Nephrol. 2009;20(11):2291-2295.

7. FDA Drug Safety Communication: low magnesium levels can be associated with long-term use of proton pump inhibitor drugs (PPIs). http://www. fda.gov/Drugs/DrugSafety/ucm245011.htm. Accessed February 3, 2011.

8. Agus ZS. Hypomagnesemia. JAm Soc Nephrol. 1999;10(7):1616-1622.

9. Santos AF, Sousa F, Rodrigues M, Ferreira C, Soares-Fernandes J, Maré R. Reversible cerebellar syndrome induced by hypomagnesemia. Neurol Clin Neurosci. 2015;3(5):190-191.

10. Sedehizadeh S, Keogh M, Wills AJ. Reversible hypomagnesaemia-induced subacute cerebellar syndrome. Biol Trace Elem Res. 2011;142(2):127-129.

11. Te Riele MG, Verrips A. Severe hypomagnesaemia causing reversible cerebellopathy. Cerebellum (London, England). 2014;13(5):659-662.

12. Leicher CR, Mezoff AG, Hyams JS. Focal cerebral deficits in severe hypomagnesemia. Pediatr Neurol. 1991;7(5):380-381.

13. Mehra R, To CY, Qahwash O, Richards B, Fessler RD, 2nd. Intravenous thrombolysis in acute ischemic stroke patients with negative CT perfusion: a case series. Acta Radiol Short Rep. 2014;3(7):2047981614543219.

14. Oppenheim C, Stanescu R, Dormont D, et al. False-negative diffusionweighted MR findings in acute ischemic stroke. AJNR Am J Neuroradiol. 2000;21(8):7.

15.Lewandowski C, Mays-Wilson K, Miller J, et al. Safety and outcomes in stroke mimics after intravenous tissue plasminogen activator administration: a single-center experience. J Stroke Cerebrovasc Dis. 2015;24(1):48-52.

16. Chang J, Teleb M, Yang JP, et al. A model to prevent fibrinolysis in patients with stroke mimics. J Stroke Cerebrovasc Dis. 2012;21(8):839-843.

17. Chernyshev OY, Martin-Schild S, Albright KC, et al. Safety of tPA in stroke mimics and neuroimaging-negative cerebral ischemia. Neurology. 2010;74(17):1340-1345.

18. The National Institute of Neurological Disorders and Stroke rt-PA Stroke Study Group. Tissue plasminogen activator for acute ischemic stroke. N Engl J Med. 1995;333(24):1581-1587.
International Medical Case Reports Journal

\section{Publish your work in this journal}

The International Medical Case Reports Journal is an international, peer-reviewed open-access journal publishing original case reports from all medical specialties. Previously unpublished medical posters are also accepted relating to any area of clinical or preclinical science. Submissions should not normally exceed 2,000 words or

\section{Dovepress}

4 published pages including figures, diagrams and references. The manuscript management system is completely online and includes a very quick and fair peer-review system, which is all easy to use. Visit $\mathrm{http}: / /$ www.dovepress.com/testimonials.php to read real quotes from published authors. 